\title{
Oxygen Deficiency at a COVID-19 Epicenter: A Buying-Time Solution
}

\author{
Ailan Chen ${ }^{1,3}$, Qingquan Lv², Zhifang Cai ${ }^{3}$, Yingjie Song ${ }^{3}$, Jianheng Zhang ${ }^{3,4}$, Jiaxing Xie ${ }^{3,4, *}$ \\ ${ }^{1}$ Department of Cardiology, The First Affiliated Hospital of Guangzhou Medical University, Guangzhou, China. \\ ${ }^{2}$ Medical Department, Hankou Hospital of Wuhan City, Wuhan, China. \\ ${ }^{3}$ Department of Pulmonary and Critical Care Medicine, Hankou Hospital of Wuhan City, Wuhan, China. \\ ${ }^{4}$ Department of Pulmonary and Critical Care Medicine, National Clinical Research Center for Respiratory Disease, The First Affi- \\ liated Hospital of Guangzhou Medical University, Guangzhou, China.
}

How to cite this paper: Ailan Chen, Qingquan Lv, Zhifang Cai, Yingjie Song, Jianheng Zhang, Jiaxing Xie. (2021) Oxygen Deficiency at a COVID-19 Epicenter: A Buying-Time Solution. International Journal of Clinical and Experimental Medicine Research, 5(3), 260-263.

DOI: 10.26855/ijcemr.2021.07.005

Received: March 31, 2021

Accepted: April 27, 2021

Published: May 21, 2021

*Corresponding author: Jiaxing Xie, Department of Pulmonary and Critical Care Medicine, Hankou Hospital of Wuhan City, Wuhan, China; Department of Pulmonary and Critical Care Medicine, National Clinical Research Center for Respiratory Disease, The First Affiliated Hospital of Guangzhou Medical University, Guangzhou, China. Email: jiaxingxie@126.com

\begin{abstract}
Medical oxygen is a vital part of treatment for the coronavirus. But huge numbers of COVID-19 patients in a city may crush the entire medical care system and debilitate the medical supply chain by using up the medical resources, such as oxygen. Sustainable oxygen supply has been an issue in industrialized and is still biting poorer countries battling COVID-19. We analysis the reasons of shortages of oxygen in the pandemic and present our experiences in Wuhan in order to provide an emergent handling protocol to cities under similar circumstances during the ongoing pandemic. Strategies for improving oxygen supply, expanding the use of oxygen concentrators, and algorithmizing oxygen therapy, may buy more time for both medical care providers and COVID-19 patients. Investment in oxygen infrastructure should be prioritized not only for COVID-19 but also for other unpredictable pandemic and conditions that cause hypoxaemia.
\end{abstract}

\section{Keywords}

Oxygen, COVID-19, Pandemic

As the epicenter of the COVID-19 pandemic, Wuhan was unprepared for the initial wave of attacks caused by severe acute respiratory syndrome coronavirus 2 (SARS-CoV-2) in December 2019 [1-3]. Throughout its nationwide spreading stage, approximately $60 \%$ of COVID-19 cases in China were clustered in Wuhan (http://2019ncov.chinacdc.cn/nCoV). These findings suggest that once a pandemic caused by a highly contagious and deadly pathogen like SARS-CoV-2 emerges in any city, huge numbers of patients may crush the entire medical care system and debilitate the medical supply chain by using up the medical resources and capacity of the city.

In a setting lacking effective therapeutic measures, adequate respiratory support with supplemental oxygen has been recommended by the World Health Organization as a primary and crucial intervention for people suffering from hypoxemia and striving for their survival [4]. However, as new COVID-19 cases have been surging all over the world, the demand for oxygen outpaces its supply. Shortages of oxygen supply have left patients gasping for breath, especially in developing countries $[5,6]$. Sustainable oxygen supply has also been an issue in industrialized countries [7-9]. This mainly attributed to that: 1) a large number of COVID-19 patients require oxygen therapy at the same time; 2) oxygen tank with a low storage capacity; 3) liquid oxygen vaporizer with insufficient gasification capacity; 4) oxygen pipes in many hospitals are not able to accommodate the increased flow demands due to design limitations; and 5) Logistics industry, which is involved in the movement, storage, and flow of liquid oxygen and cylinders, have been directly affected by the COVID-19 pandemic lockdown. Here, we humbly present our expe- 
riences in Wuhan in order to provide an emergent handling protocol to cities under similar circumstances during the ongoing pandemic.

Wuhan Hankou Hospital is a 430-bed tertiary comprehensive hospital located in the center of Wuhan, less than 4 km away from the Huanan Seafood Market, and its pre-COVID-19 oxygen storage and delivery capacities had been designed as one liquid medical oxygen tank with a capacity of $5 \mathrm{~m}^{3}$ with the maximum oxygen supply flow rate of the evaporator as $50 \mathrm{~m}^{3}$ per hour. As one of three designated COVID-19 hospitals identified by the government of Wuhan on January 21, the number of COVID-19 patients surged and peaked on February 22 in our hospital, cause low oxygen flow from all the wall oxygen outlets. Because of the barely optimized reality within our hospital when COVID-19 suddenly came down from nowhere, several buying-time measures were developed and implemented in January and February 2020 to effectively deliver sufficient oxygen to patients.

To reduce the draw on the wall oxygen in hospitals, portable oxygen cylinder was used widely in Wuhan at the beginning of pandemic, however, the increased use of portable oxygen cylinder is contributing to a shortage of oxygen cylinders of all sizes. Timely oxygen cylinders withdraw from hospitals, disinfection and delivery to hospitals has also been a problem [10], and oxygen flow regulators, which are needed for both wall oxygen and portable oxygen tanks, are in critically short supply. During the initial stage of the pandemic, thousands of oxygen cylinders were made from other districts and then distributed to these designated hospitals in Wuhan. Logistics industry increased manpower to transport oxygen cylinders. In our hospital, the oxygen supply increased from 29 cylinders to 150 cylinders each day during the COVID-19 pandemic. But changing cylinders was labor consuming for medical providers.

Oxygen concentrators allow us to extract oxygen from the air using a sieving process and providing oxygen with purity $>90 \%$ at a variety of flow outputs [11], which can provide sufficient oxygen to most patients. Compared with oxygen cylinders, oxygen concentrators are simpler and safer to operate and can be readily adapted for use in non-oxygen equipped extra beds, casualty wards, nursing homes, community clinics without a central oxygen supply. These oxygen concentrators do not need oxygen resupply or flow regulators.

In Wuhan, some hospitals have added secondary liquid oxygen dewars, and vaporizers. Some hospitals have improvised warm water sprinkler systems or warm air heating system to keep the external vaporizers from freezing. A few hospitals have other solutions, including connecting directly to trucks carrying bulk liquid oxygen, installing large oxygen generators, secondary oxygen supply lines that bypass the existing oxygen delivery systems. But installing new oxygen pipes system is both time and labor consuming. Huo Shen Shan Hospital and Lei Shen Shan Hospital-two newly built specialized hospitals in Wuhan for the centralized treatment of COVID-19 patients-were designed and equipped with medical oxygen supply systems with much higher capacity, based on the oxygen issues of the above hospitals are facing.

Following our arrival at Hankou Hospital, tailored guideline (Figure 1) for oxygen therapy were developed for the COVID-19 wards. The goal of this guideline is to support the reasonable use of oxygen in cases where ICU beds are overwhelmed and there is insufficient oxygen supply, to maximize the oxygen supply to critically ill patients, and to prolong their lives until they have the opportunity to transfer to ICU.

1. Basically, hundreds of oxygen concentrators were used as much as possible in place of oxygen cylinders at our hospital, dramatically reducing workload and improving safety.

2. In most patients with severe COVID-19, 2 concentrators (one connects to nasal flow and another connects to reservoir masks) were used on a single patient to provide higher oxygen concentrations and could replace wall outlet-oxygen to reduce the burden on the central oxygen supply.

4. Oxygen concentrators could also be used in combination with other oxygen sources (e.g., oxygen masks connected to the wall outlet + nasal cannula connected to an oxygen concentrator; oxygen masks connected to oxygen cylinders + nasal cannula connected to an oxygen concentrator), which yielded a total oxygen flow of up to 10-15 $\mathrm{L} / \mathrm{min}$. In particular, additional nasal cannula connected to an oxygen concentrator was given to patients on non-invasive ventilators. Dual oxygen therapy improves oxygenation in critically ill patients and earns time for the patient to be intubated and transferred to ICU.

5. A large number of pulse oximeters were rapidly issued to Hankou Hospital during the outbreak. Oxygen flow from wall outlets was titrated to achieve a target oxygen saturation of 93\% in COVID-19 patients, to reduce the burden on the central oxygen supply. Evidence from clinical trials suggests that lower oxygen target ranges are safe $[12,13]$. All doctors and nurses were trained not to waste oxygen. Once the condition of a patient with COVID-19 had improved (oxygen saturation 93\%), an oxygen concentrator was substituted for the connection to wall outlets, 
in order to continue oxygen therapy without reducing consumption of the central oxygen supply, which was preserved for either high-flow nasal cannulas (HFNC), or non-invasive ventilators used to treat severely ill patients.

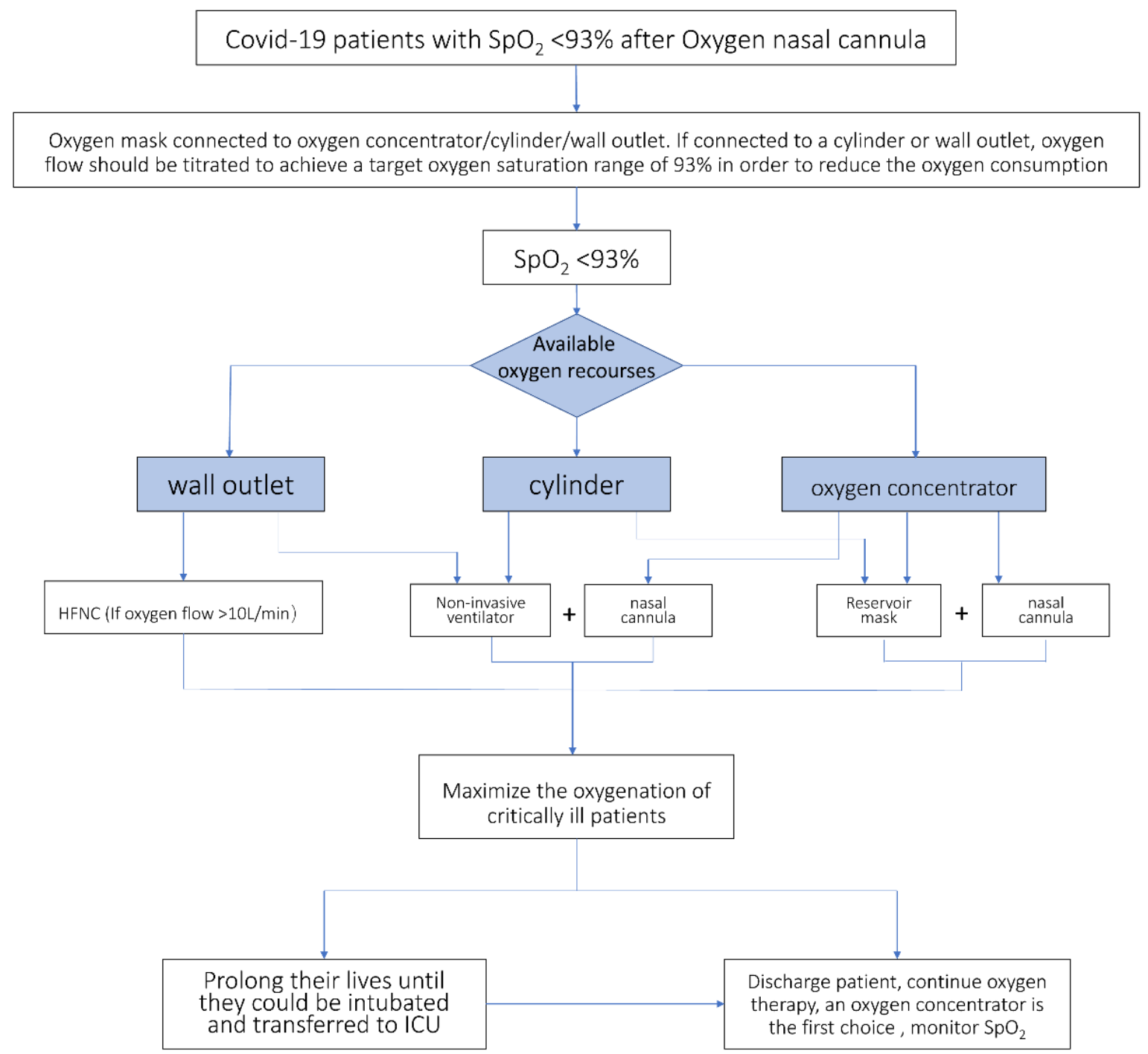

Figure 1. A buying-time solution to oxygen deficiency in one of the covid-19 pandemic epicenters.

\section{Conclusion}

In conclusion, during a sudden outbreak of the COVID-19 pandemic, supplemental oxygen supply shortage is a crucial issue, even in a modern hospital. In our experience, strategies for improving oxygen supply, expanding the use of oxygen concentrators, and algorithmizing oxygen therapy, may buy more time for both medical care providers and COVID-19 patients. Hospital systems, health authorities, state officials, and the federal government in the world should prioritize investment in oxygen infrastructure, not only for COVID-19 and severe acute respiratory infections but also for other unpredictable pandemic and conditions that cause hypoxaemia.

\section{Declaration of interests}

All the authors declare no competing interests.

\section{Funding/Support}

This study is supported by the Guangdong Province Science and Technology Innovation Strategy Special Foundation (Grant No. 2020B111134019) and Zhongnanshan Medical Foundation of Guangdong Province. 


\section{Ethic approval}

This study was approved by the ethics committee of Hankou Hospital.

\section{Contributors}

Jiaxing Xie, Ailan Chen and Qingquan Lv contributed to the study design and methodology. Zhifang Cai, Yingjie Song and Jianheng Zhang were responsible for the oversight of the study. Jiaxing Xie and Ailan Chen wrote the first draft of the manuscript. All authors contributed to critical revision of the manuscript for important intellectual content and gave final approval.

\section{References}

[1] Wu, Z., McGoogan, J. M. (2020). Characteristics of and Important Lessons From the Coronavirus Disease 2019 (COVID-19) Outbreak in China: Summary of a Report of 72314 Cases From the Chinese Center for Disease Control and Prevention. JAMA, 323: 1239-1242.

[2] Bi. Q., Wu. Y., Mei. S., Ye. C., Zou. X., Zhang. Z., Liu. X., Wei. L., Truelove. S. A., Zhang, T., et al. (2020). Epidemiology and transmission of COVID-19 in 391 cases and 1,286 of their close contacts in Shenzhen, China: a retrospective cohort study. Lancet Infect Dis, 20: 911-919.

[3] Guan, W. J., Ni, Z. Y., Hu, Y., Liang, W. H., Ou, C. Q., He, J. X., Liu, L., Shan, H., Lei, C. L., Hui, D. S. C., et al. (2020). Clinical Characteristics of Coronavirus Disease 2019 in China. N Engl J Med., 382: 1708-1720.

[4] World Health Organization. (2020). Oxygen sources and distribution for COVID-19 treatment centres.

[5] Stein, F., Perry, M., Banda, G., Woolhouse, M., Mutapi, F. (2020). Oxygen provision to fight COVID-19 in sub-Saharan Africa. BMJ Glob Health, 5.

[6] World Health Organization. (2021). COVID-19 oxygen emergency impacting more than half a million people in low- and middle-income countries every day, as demand surges.

[7] The Guardian. (2020). Coronavirus: London hospital almost runs out of oxygen for Covid-19 patients.

[8] FORTUNE. (2020). Coronavirus treatment: Hospitals face supply shortage for COVID-19 patients.

[9] Eric, T. (2021). Potential Solutions to the COVID-19 Oxygen Crisis in the United States.

[10] Ritz, R. H., Previtera, J. E. (2008). Oxygen supplies during a mass casualty situation. Respir Care, 53: 215-224; discussion 224-215.

[11] Bolton, C. E., Annandale, J. A., Ebden, P. (2006). Comparison of an oxygen concentrator and wall oxygen in the assessment of patients undergoing long term oxygen therapy assessment. Chron Respir Dis., 3: 49-51.

[12] Siemieniuk, R. A. C., Chu, D. K., Kim, L. H., Güell-Rous, M. R., Alhazzani, W., Soccal, P. M., Karanicolas, P. J., Farhoumand, P. D., Siemieniuk, J. L. K., Satia, I., et al. (2018). Oxygen therapy for acutely ill medical patients: a clinical practice guideline. BMJ, 363:k4169.

[13] Chu, D. K., Kim, L. H., Young, P. J., Zamiri, N., Almenawer, S. A., Jaeschke, R., Szczeklik, W., Schünemann, H. J., Neary, J. D., Alhazzani, W. (2018). Mortality and morbidity in acutely ill adults treated with liberal versus conservative oxygen therapy (IOTA): a systematic review and meta-analysis. Lancet, 391: 1693-1705. 